\title{
Combined treatment with Octreotide LAR and Pegvisomant in patients with Acrogigantism: Clinical Evaluation and Genetic Screening
}

\author{
Liliya Rostomyan 1 , Ruth Mangupli3 ${ }^{3}$ Emilie Castermans², Jean-Hubert Caberg², Paul Camperos ${ }^{3}$, \\ Jaime Krivoy ${ }^{3}$, Elvia Cuauro ${ }^{3}$, Vincent Bours ${ }^{2}$, Adrian F Daly ${ }^{1}$, Albert Beckers ${ }^{1}$ \\ (1) Department of Endocrinology and (2) Department of Clinical Genetics, Centre Hospitalier Universitaire de Liège, University \\ of Liège, Liège, Belgium (3) Section of Neuroendocrinology, Department of Neurosurgery, Hospital Universitario de \\ Caracas, Caracas, Venezuela
}

Pituitary gigantism is a rare condition caused by growth hormone hypersecretion, usually by a pituitary tumor. Acromegaly and gigantism cases that have a genetic cause are challenging to treat, due to large tumor size and poor responses to some medical therapies (e.g. AIP mutation affected cases and those with X-linked acrogigantism (X-LAG) syndrome.

\section{Objectives}

We studied a gigantism cohort from Venezuela for genetic defects and their response to treatment.
We performed a retrospective study to identify gigantism cases among 160 somatotropinoma patients treated between 1985 and 2015 at the University Hospital of Caracas, Venezuela. We studied clinical details at diagnosis, hormonal responses to therapy and undertook targeted genetic testing that included AIP and MEN1 sequencing and MLPA, and aCGH for Xq26.3 duplications.

\section{Results}

Among the 160 cases, 8 patients ( 6 males; $75 \%$ ) were

diagnosed with acrogigantism, all developed their $1^{\text {st }}$ clinical symptoms before 21 years. The most frequent clinical signs at presentation (apart from tall stature) were acral enlargement (7/8) and headache (5/8). All patients had $\mathrm{GH}$ secreting pituitary macroadenomas (Median max diameter 31mm, IQR: 25.5-41.5) with cavernous sinus invasion in $75 \%$ and prolactin hypersecretion in $50 \%$. Six cases received primary treatment with the long-acting SSA octreotide LAR $20 \mathrm{mg} / 28$ days for $6-12$ months, in 2 cases SSA was

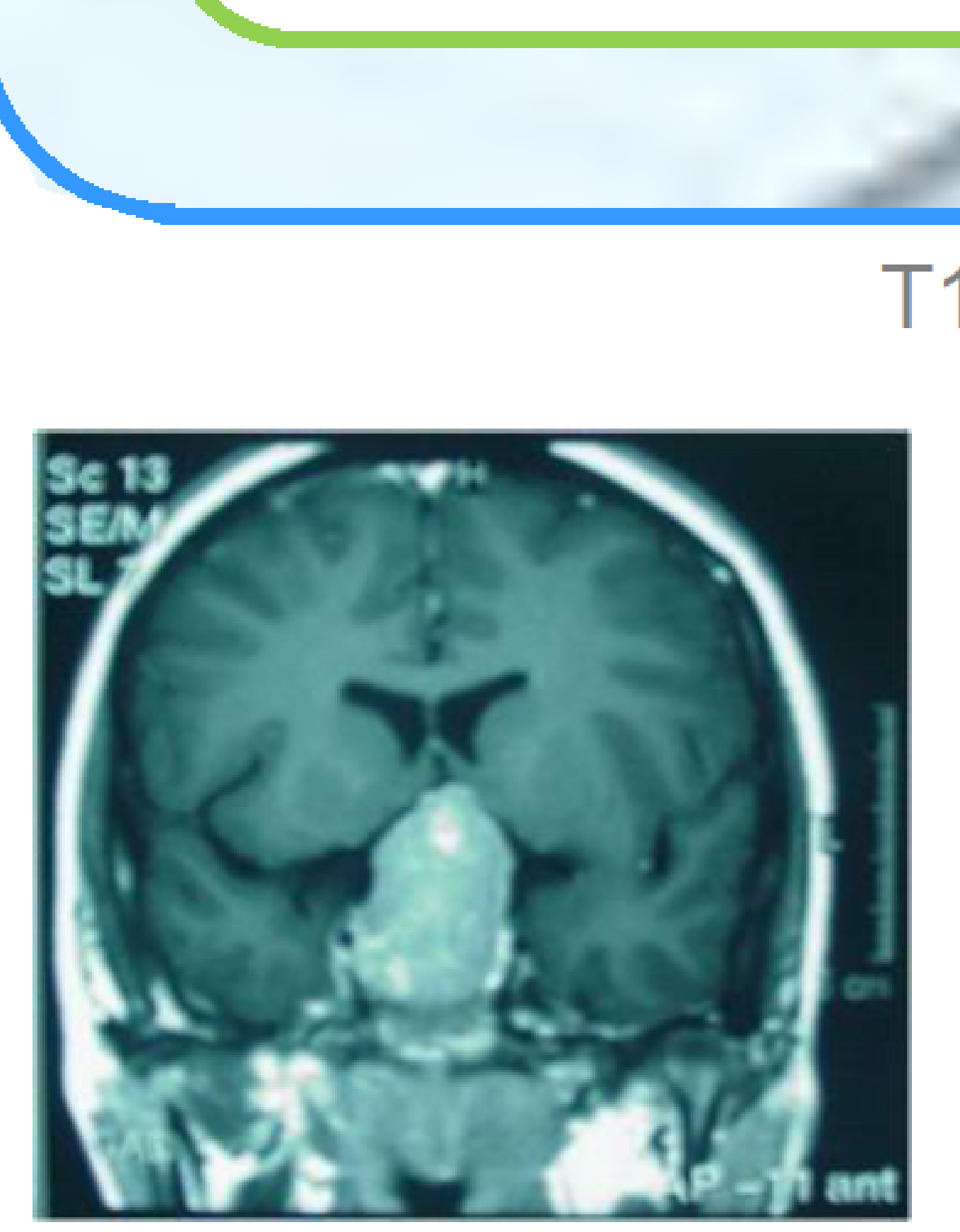

Patient \#1

T1-weighted coronal MR imaging

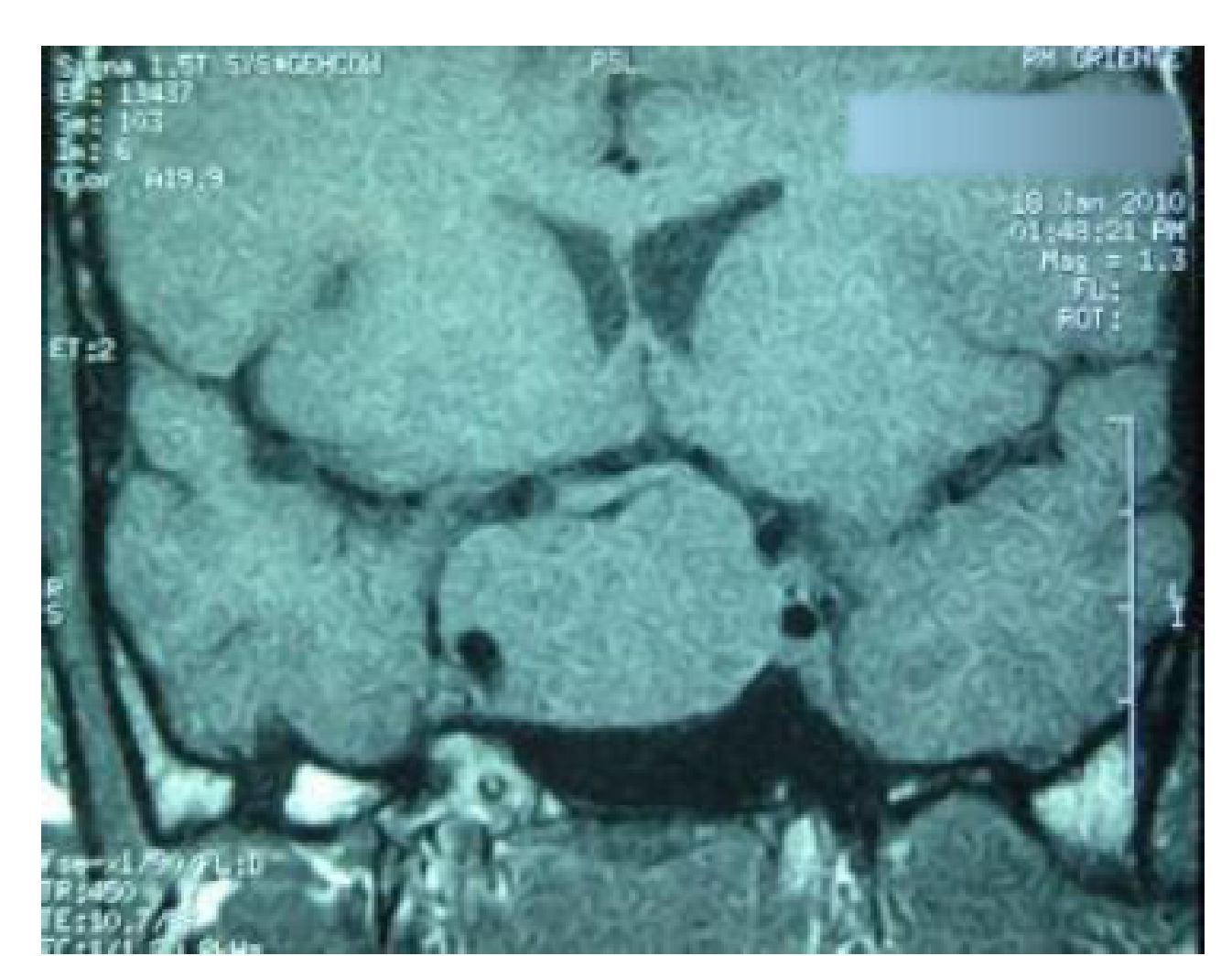

Patient \#5

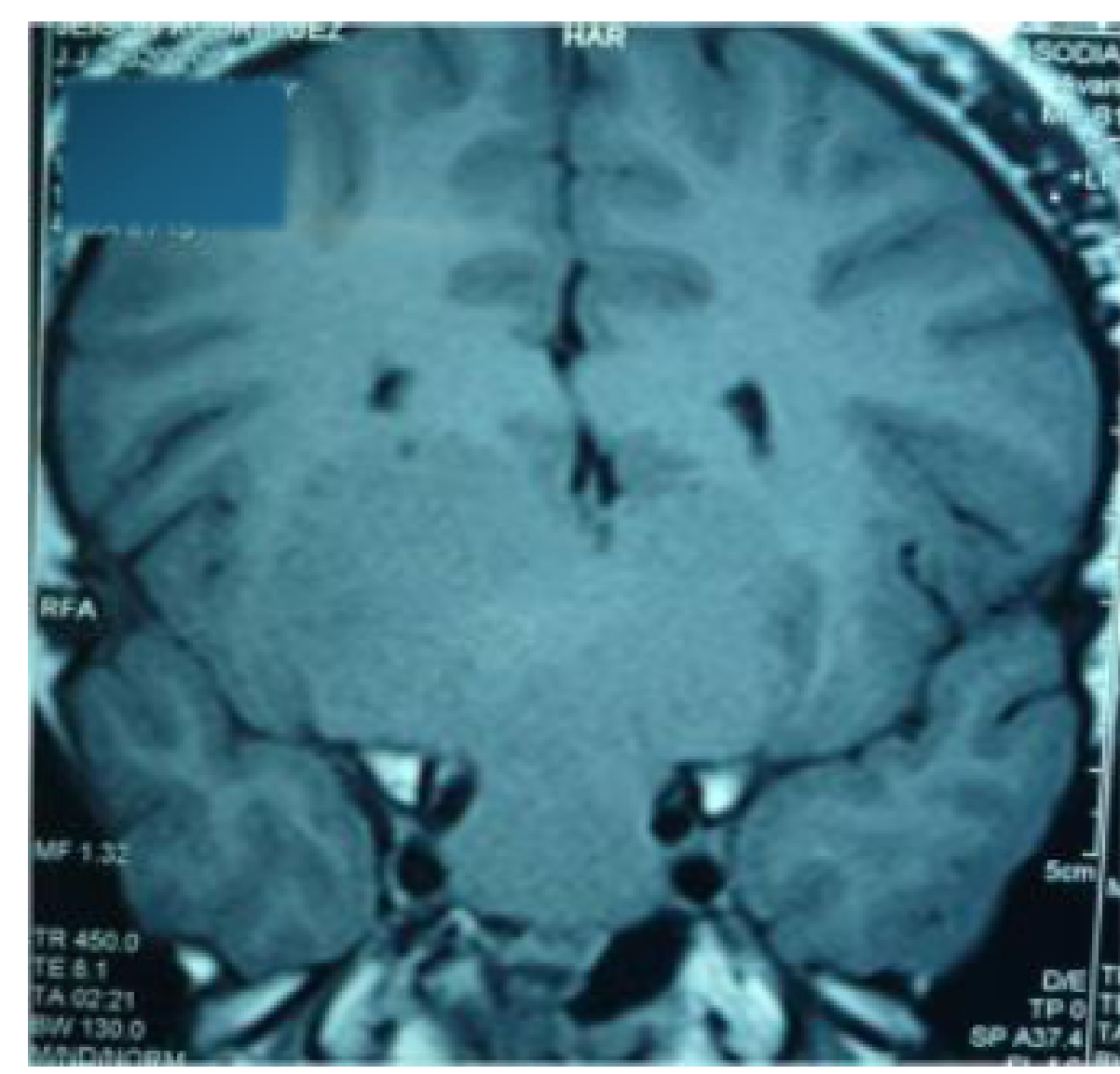

Patient \#7

administrated after unsuccessful surgery (radiotherapy was also used in 1 case). Cabergoline was added in those with elevated prolactin. None of the patients had hormonal control. Additional administration of Pegvisomant $20 \mathrm{mg}$ daily resulted in a decrease of IGF-1 to normal ranges while tumor volume was stable in all patients. Regression of clinical symptoms was seen after 1-4 months of treatment including a decrease in growth velocity. None of the patients had evidence of MEN1, Carney complex, FIPA or McCune Albright syndromes. Novel AIP mutations were the found in three patients. None of the patients had MEN1 mutations/deletions or Xq26.3 microduplications.

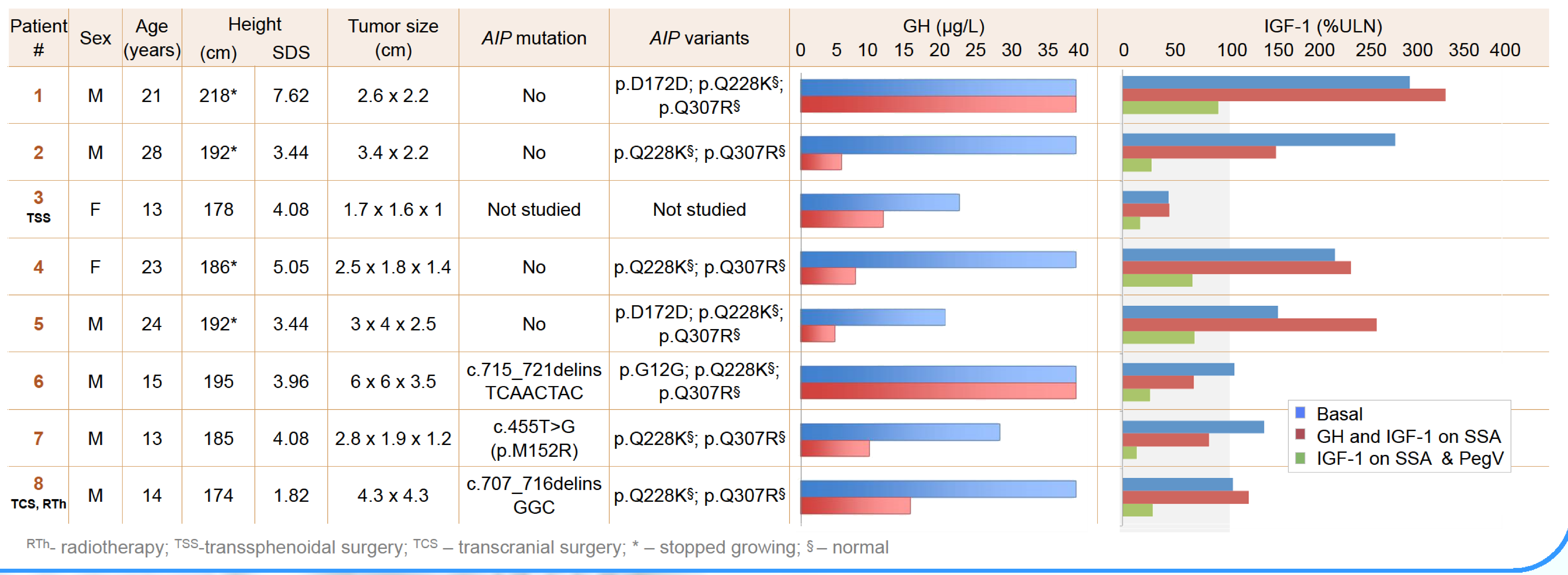

\section{Conclusions}

Patients with gigantism have large and aggressive GH secreting pituitary lesions that are difficult to control with conventional treatment options. Prolactin co-secretion is frequent. Treatment of acrogigantism is

frequently challenging; delayed control increases the harmful effects of $\mathrm{GH}$ excess, such as, excessive stature and symptom burden. Combined therapy (long-acting SSA and pegvisomant) as primary treatment or after pituitary surgery and radiotherapy can permit the normalization of IGF-1 levels and achieve clinical improvement in these difficult to manage patients. 